\title{
Spontaneous Posterior Spinal Cord Infarction Presenting as Sensory Disturbance with Radiating Pain
}

\author{
Junghee Seo* (D), Mun Kyung Sunwoo* (D), Hee Jin Lee ${ }^{*}$ (D), Young Ik Jung ${ }^{*}$ (D), Jeongyeon Kim ${ }^{\dagger}$ (D) \\ Department of Neurology, Bundang Jesaeng General Hospital ${ }^{\star}$, Seongnam; Department of Neurology, Sanggyepaik Hospital, Inje University ${ }^{\dagger}$, \\ Seoul, Korea
}

Cervical posterior spinal artery (PSA) infarction is rare. We report a case of PSA syndrome that presented as neck and shoulder pain followed by unilateral weakness and sensory disturbance. Motor and sensory symptoms were related to the lateral corticospinal tract and posterior column, respectively. Radiating pain in the neck and shoulder might be associated with the dorsal horn, which integrates sensory circuits. This is a case of PSA infarction presenting as sensory disturbance with radiating pain associated with dorsal horn involvement.

J Neurosonol Neuroimag 2019;11(2):17 $\$-1 \$ 1$

Key Words: Infarction; Spinal cord; Spinal cord dorsal horn; Pain
Received: November 7, 2019

Revised: November 29, 2019 Accepted: December 4, 2019

Address for correspondence: Jeongyeon Kim

Department of Neurology, Sanggyepaik Hospital, Inje University, 1342 Dongil-ro, Nowon-gu, Seoul 01757, Korea Tel: +82-2-950-1090 Fax: $+82-2-950-1955$ E-mail:jykimmd@paik.ac.kr
Spinal cord infarction (SCI) is a rare disease that accounts for approximately $1.2 \%$ of all strokes. ${ }^{1,2}$ Because the clinical presentation, severity, and outcomes of SCI vary widely, under-diagnosis is highly probable. ${ }^{3,4}$ SCI usually occurs in the territories of the anterior spinal artery (ASA). ASA syndrome typically manifests as a sudden onset of bilateral weakness, flaccid paraplegia, areflexia, and dissociative anesthesia. ${ }^{2,5,6}$ However, posterior spinal artery (PSA) infarctions are very rare and thus, reports on the clinical and radiological features of PSA syndrome are lacking. We report a case of unilateral PSA syndrome, which first presented as neck and shoulder pain followed by unilateral weakness and sensory disturbance.

\section{CASE REPORT}

An 86-year-old male with a history of hypertension, depression, and right total knee replacement (TKR) visited our hospital with acute onset of right hemiplegia and numbness accompanied by shoulder pain. One day before the weakness occurred, he experienced severe pain in the right side of the neck and shoulder. He developed right side paralysis with worsening shoulder pain; thus, he visited the emergency room one day after onset. Postoperative sequelae after TKR were not observed and the patient reported no history of trauma. Initial examination revealed that he was alert and oriented. His muscle strength was graded three out of five in the right upper extremity and one out of five in the right lower extremity. Sensory disturbance in proprioception and vibratory sensation on the right side of his body were noticed. Pain and temperature sensation were preserved on both sides. However, paresthesia was observed in the right shoulder and lower neck area. Deep tendon reflexes in the right brachioradialis and biceps were absent. Laboratory data including complete blood cell count, erythrocyte sedimentation rate, $\mathrm{C}$-reactive protein, and liver and renal function tests were unremarkable. However, he had high fasting serum glucose (134 mg/dL) and low-density lipoprotein (159 mg/dL) levels. There was no evidence of systemic vasculitis. Non-contrast enhanced computed tomography, which definitively excluded parenchymal hemorrhage, was normal. Diffusion-weighted magnetic resonance imaging (MRI) revealed definitive acute infarction in brain parenchyma; however, a slightly 
high signal intensity was observed in the upper cervical spinal cord. Cervical cord MRI revealed high intensity areas at the $\mathrm{C}_{2}-\mathrm{C}_{4}$ levels of the right posterior cervical cord including the posterior column, lateral corticospinal tract, and dorsal horn (Fig. 1) on diffusion-weighted images accompanied by subtle T2 signal changes. Magnetic resonance angiography showed multiple stenosis and irregularities in the right vertebral artery $\mathrm{V}_{4}$ segment. To differentiate between simple stenosis and dissection, transfemoral cerebral angiography was performed. This revealed the absence of an intimal flap, a double lumen sign, and pearls sign, indicating dissection (Fig. 2). Echocardiogram showed no evidence of intracardiac thrombus. Finally, cervical cord infarction due to atherosclerosis was diagnosed, anti-platelet agents (aspirin $100 \mathrm{mg}$, plavix $75 \mathrm{mg}$ ) were prescribed, and bedside rehabilitation begun. The right side muscle weakness did not improve despite regular rehabilitation. Finally, he passed away from aspiration pneumonia as a complication of cervical spinal cord infarction.

\section{DISCUSSION}

The PSA supplies the posterior one third of the spinal cord including the posterior column, posterior horn, and posterior segments of the lateral column. PSAs are fed by bilateral vertebral arteries and several posterior segmental radicular arteries and anastomose with the ASA around the conus medullaris. Because of this network, infarctions in the PSA region are rare. ${ }^{3}$ In addition, the area supplied by PSA does not have a clear boundary; thus, there are a broad range of clinical manifestations of PSA infarction depending on the lesions involved. ${ }^{3,7}$ Typically, patients with PSA infarct present with abrupt sensory ataxia due to proprioceptive dysfunction. ${ }^{3,4,7}$ Unilateral motor weakness is considered an atypical symptom of PSA infarction, because the ASA supplies the lateral funiculus including the pyramidal tract. ${ }^{7}$ However, clinical symptoms are not only limited to posterior column function but are heterogeneous. In previous studies, pyramidal tract involvement was reported to be more common than originally thought. ${ }^{4,7}$ Sakurai et al. ${ }^{7}$ reported that paralysis was frequently observed in patients with cervical PSA syndrome compared to a small number of patients without pyramidal

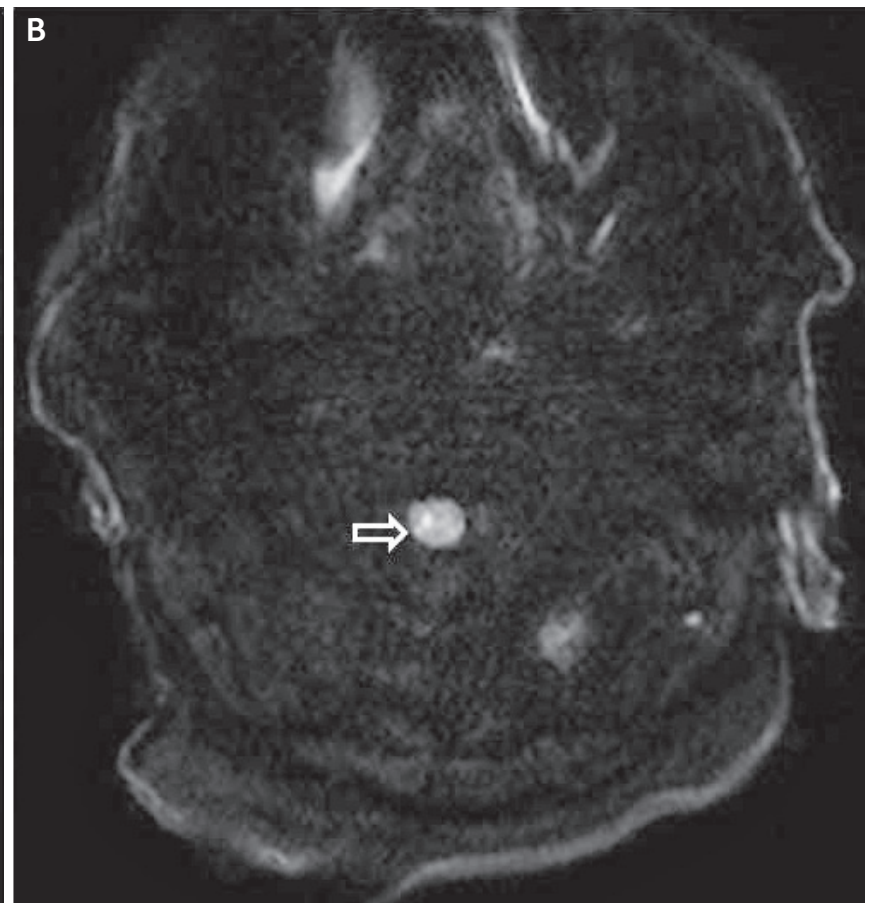

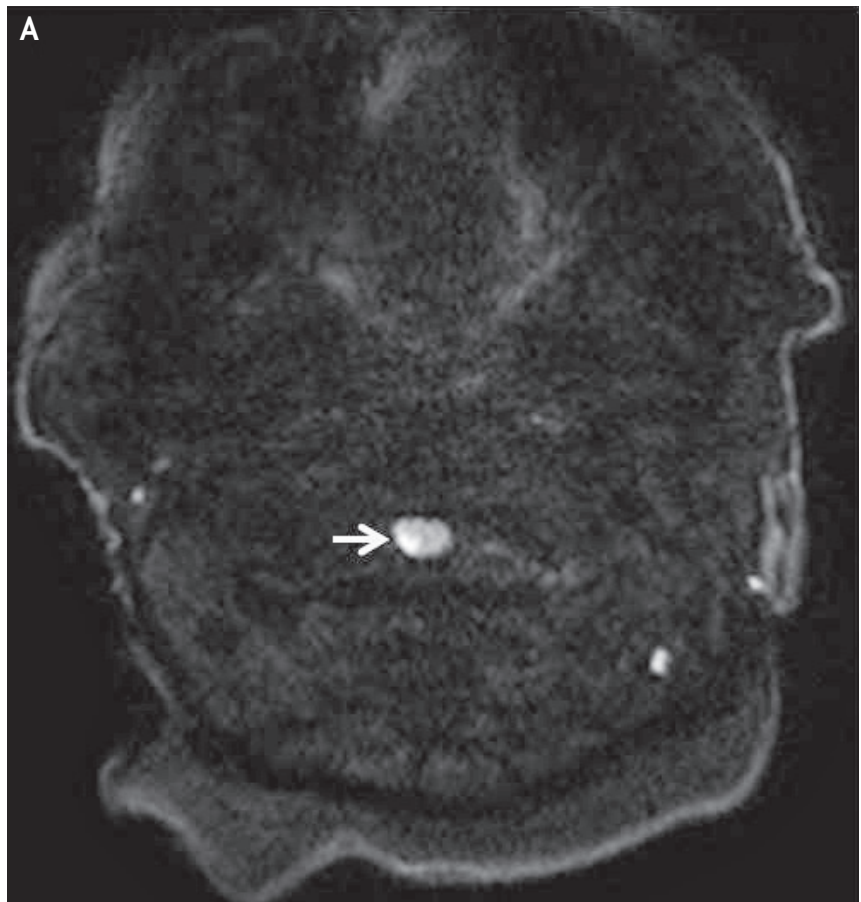

FIG. 1. Cervical cord magnetic resonance imaging revealed high intensity areas at the right posterior cervical cord including the posterior column, (A) lateral corticospinal tract (arrow), and (B) dorsal horn (arrow) on diffusion-weighted images. 

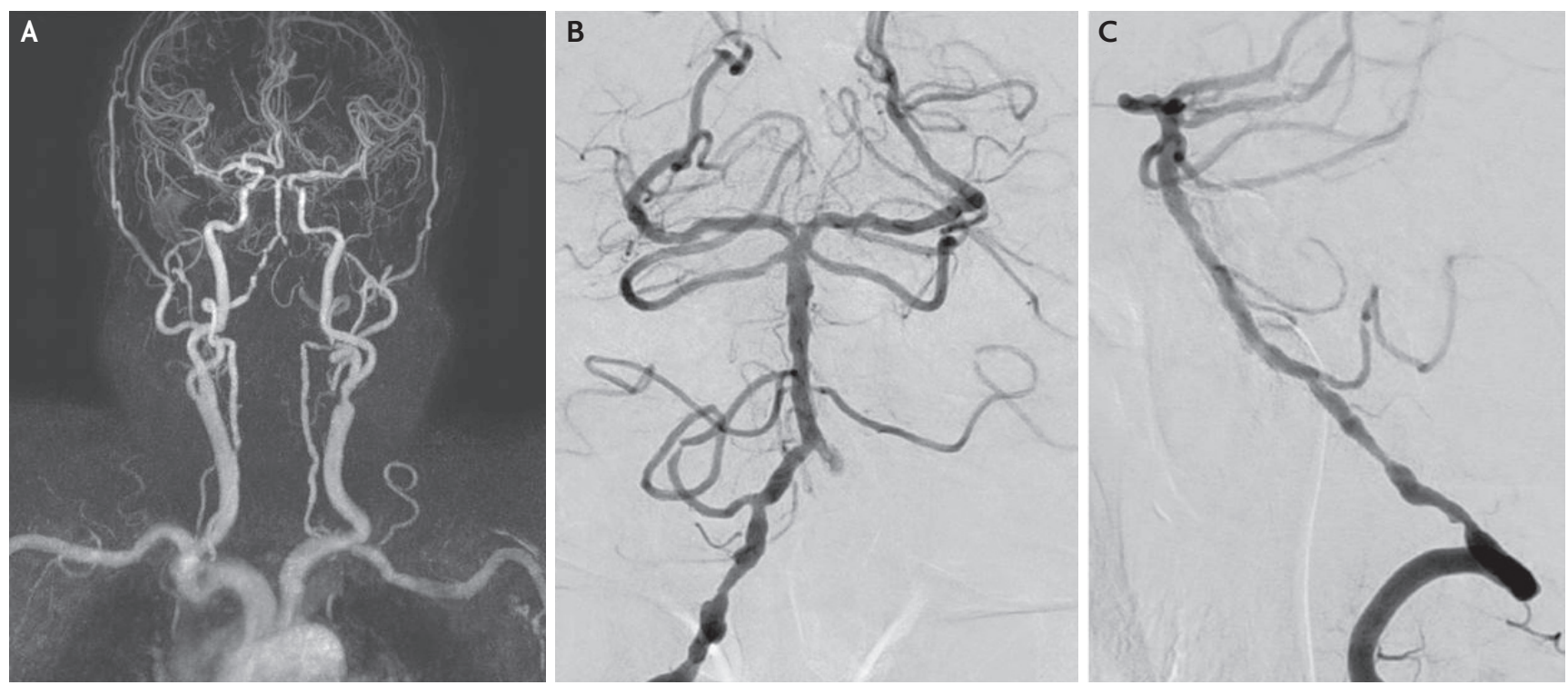

FIG. 2. (A) Magnetic resonance angiography revealed multiple atherosclerotic stenosis. However, (B, C) transfemoral cerebral angiography showed no evidence of dissection such as intimal flap, double lumen sign, and pearls sign.

signs. In another case report, patients with PSA infarction showed mild-to-severe motor deficits. According to a recent Mayo Clinic report, $73 \%$ of 15 patients with PSA infarction showed unilateral motor weakness. ${ }^{4}$ In our case, the patient also presented with a moderate degree of right side weakness, confirming the high frequency of motor involvement that was previously reported.

In our patient, pain was also one of the symptoms that needed to be addressed. Initially, the vertebral artery dissection was suspected to be the cause of the PSA infarction, due to the radiating pain in the posterior neck and right shoulder. However, evidence of dissection could not be confirmed based on radiological study and therefore, atherosclerotic change was considered to be the cause. Patients with PSA infarction are frequently reported to experience pain that often radiates and is associated with the ischemic spinal level., ${ }^{3,4}$ Struhal et al. ${ }^{3}$ reported that three out of four patients experienced a sudden onset of pain localized to a specific sensory level. In another PSA infarction study, 60\% of the patients presented with an abrupt sensory disturbance accompanied by pain. ${ }^{4}$ The exact pathophysiology of pain associated with PSA syndrome was not elucidated; however, the pain was suggested to be associated with neuronal circuitry processing in the dorsal horn. ${ }^{8,9}$ Dorsal horn neurons in the spinal cord receive sensory information from peripheral afferent nerves. The incoming information is processed by complex circuits in the dorsal horn, and transmitted to projection neurons for relay to the brain. ${ }^{9}$ Although our knowledge of dorsal horn circuitry remains limited, nerve injury or inflammation of the circuits in the dorsal horn results in a heightened response to incoming afferent signals and increased output to the brain. Therefore, this sensory signal interruption contributes to the exaggerated feeling of pain..$^{8-10}$ In our patient, not only the right posterior column, but also the right dorsal horn in the upper cervical region, was involved. Therefore, the processing of pain might have been disturbed, resulting in radiating pain in the neck and right shoulder.

In conclusion, we reported a case involving a patient with PSA infarction who presented with right side sensory change, motor weakness, and radiating pain. The lesions associated with the PSA infarction involved the posterior column, lateral corticospinal tract, and dorsal horn. Motor and sensory symptoms were associated with the lateral corticospinal tract and posterior column, respectively. Radiating pain in the neck and shoulder might be associated with the dorsal horn, which integrates sensory circuits. Although myelopathy is a common cause of spinal cord lesions, in cases involving the abrupt onset of symptoms, spinal cord infarction should be suspected. 


\section{Conflicts of Interest}

No potential conflicts of interest relevant to this article was reported.

\section{REFERENCES}

1. Sandson TA, Friedman JH. Spinal cord infarction. Report of 8 cases and review of the literature. Medicine (Baltimore). 1989;68:282-292.

2. Salvador de la Barrera S, Barca-Buyo A, Montoto-Marqués A, Ferreiro-Velasco ME, Cidoncha-Dans M, Rodriguez-Sotillo A. Spinal cord infarction: prognosis and recovery in a series of 36 patients. Spinal Cord. 2001;39:520-525.

3. Struhal W, Seifert-Held T, Lahrmann H, Fazekas F, Grisold W. Clinical core symptoms of posterior spinal artery ischemia. Eur Neurol. 2011;65:183-186.

4. Zalewski NL, Rabinstein AA, Wijdicks EFM, Petty GW, Pittock SJ, Mantyh WG, et al. Spontaneous posterior spinal artery infarction: an under-recognized cause of acute myelopathy. Neurology. 2018;91:414-417.

5. Masson C, Pruvo JP, Meder JF, Cordonnier C, Touzé E, De La Sayette V, et al. Spinal cord infarction: clinical and magnetic resonance imaging findings and short term outcome. J Neurol Neurosurg Psychiatry. 2004;75:1431-1435.

6. Yadav N, Pendharkar H, Kulkarni GB. Spinal cord infarction: clinical and radiological features. J Stroke Cerebrovasc Dis. 2018;27:2810-2821.

7. Sakurai T, Wakida K, Nishida H. Cervical posterior spinal artery syndrome: a case report and literature review. J Stroke Cerebrovasc Dis. 2016;25:1552-1556.

8. D'Mello R, Dickenson AH. Spinal cord mechanisms of pain. Br J Anaesth. 2008;101:8-16.

9. Todd AJ. Neuronal circuitry for pain processing in the dorsal horn. Nat Rev Neurosci. 2010;11:823-836.

10. Koch SC, Acton D, Goulding M. Spinal circuits for touch, pain, and itch. Annu Rev Physiol. 2018;80:189-217. 